\section{Dynamics of Ethanolic Intoxications between the 15th of March 2020 to the 15th of March 2021 in the Context of the Covid-19 Pandemic}

\section{Mihai TERPAN ${ }^{1}$, Anamaria CIUBARA ${ }^{2}$}

${ }^{1} \mathrm{PhD}$ student, 'Dunărea de Jos' University, Galați, Romania, terpan.mihai@yahoo.com

2 University Professor, Head of the Department of Psychiatry, 'Dunărea de Jos' University, Galați, Romania; Primary psychiatrist, 'Elisabeta Doamna' Psychiatric Hospital Galați, Romania, anamaria.ciubara@ugal.ro
Abstract: In Romania, the consumption of alcoholic beverages has been and still is a cultural mechanism for socializing and reducing anxiety, regardless of age and gender. This paper evaluates the trends related to people diagnosed with acute intoxication (F10.0) and harmful use (F10.1) at the 'Elisabeta Doamna' Psychiatriy Hospital in Galati, during the pandemic, in the period between the 15th of March 2020 and the 15th of March 2021. We analysed the data base of discharges from the 'Elisabeta Doamna' Psychiatry Hospital from the 15th of March 2020 to the 15th of March 2021. ICD-10 (Classification of mental and behavioural disorders) was used for diagnosis of psychiatric disorders. We selected outpatients with the codes for acute intoxication (F10.0) and harmful use (F10.1) and excluded all patients with other psychiatric diagnoses. The data were statistically processed using: Microsoft Office-Excel, The jamovi project (2021) jamovi (Version 1.6) [Computer Software]. During the analysed period, there were 7614 discharges from the 'Elisabeta Doamna' Psychiatriy Hospital of which 1465 (20.08\%) disorders were related to alcohol consumption. Disorders related to intoxication and alcohol use represent $13.14 \%$ (957 cases) of the total discharges and $65.46 \%$ of the total number of disorders related to alcohol. Disorders related to harmful use (F10.1) represent $30.31 \%$ (442 cases), and a percentage of $35.15 \%$ (515 cases) with disorders due to acute intoxication (F10.0) out of the total cases were related to alcohol consumption. From the view of the dispersion of the number of cases, there is a fluctuation in the tendency to follow the restrictions imposed by the authorities.

Keywords: Alcohol, Covid-19, pandemic, psychiatry, intoxication, harmful use.

How to cite: Terpan, M., \& Ciubara, A. (2021). Dynamics of Ethanolic Intoxications between the 15th of March 2020 to the 15th of March 2021 in the Context of the Covid-19 Pandemic. BRAIN. Broad Research in Artificial Intelligence and Neuroscience, 12(2), 349-357. https://doi.org/10.18662/brain/12.2/213 


\section{Introduction}

In Romania, the consumption of alcoholic beverages has been and still is a cultural mechanism for socializing and reducing anxiety, regardless of age and gender (Luca, Baroiu et al, 2020). This is due to the easy use of beverages (accepted in the social context) (Sandu, 2020a), alcohol being found among the substances that are legally accessible at this time, alongside with nicotine and caffeine (Ciubara et al., 2015; Ciubara et al., 2018). Excessive consumption affects the normal functioning of the body, generating a multitude of diseases (Sandu 2020b; 2020c), including mental disorders (Luca, Ciubara et al., 2020).

\section{Objective}

This paper tries to evaluate the trends related to people diagnosed with acute intoxication (Tabian et al. 2021) code (F10.0) and harmful use code (F10.1) (American Psychiatric Association. 2013) hospitalized at the 'Elisabeta Doamna' Psychiatriy Hospital in Galati, during the COVID-19 pandemic in the period between the $15^{\text {th }}$ of March 2020 and the $15^{\text {th }}$ of March 2021.

The two diagnoses of acute ethanolic intoxication code (F10.0) and harmful alcohol use code (F10.1) were chosen from alcohol-related disorders, because they reflect acute consumption and associated dissociative behavioural disorders that cause a social reaction.

\section{Method}

We analysed the database of discharges from the 'Elisabeta Doamna' Psychiatriy Hospital using the temporary selection criteria in the period between the $15^{\text {th }}$ of March 2020 and the $15^{\text {th }}$ of March 2021.

We selected the discharged persons with the codes acute ethanol intoxication (F10.0) and harmful alcohol use (F10.1) and we excluded all patients with other psychiatric diagnoses.

ICD-10 (Classification of mental and behavioural disorders) was used for diagnosis of psychiatric disorders (World Health Organization. 2016).

The data were statistically processed using:

- Microsoft Office-Excel, Software]

- The jamovi project (2021). jamovi (Version 1.6) [Computer

- SOFA - Statistics Open For All version 1.5.4 


\section{Results}

\subsection{Distribution according to location and diagnosis}

Table 1. Distribution by location and Diagnosis

\begin{tabular}{ccccccc}
\hline Environment & \multicolumn{3}{c}{ F10.0 } & \multicolumn{3}{c}{ F10.1 } \\
\hline & Freq & Col $\%$ & Row $\%$ & Freq & Col \% & Row $\%$ \\
Rural & 177 & $34.4 \%$ & $55.5 \%$ & 142 & $32.1 \%$ & $44.5 \%$ \\
Urban & 338 & $65.6 \%$ & $53.0 \%$ & 300 & $67.9 \%$ & $47.0 \%$ \\
TOTAL & 515 & $100.0 \%$ & $53.8 \%$ & 442 & $100.0 \%$ & $46.2 \%$ \\
\hline
\end{tabular}

Source: Authors' own conception

Out of the total of 757 cases in the analysed period, for the diagnosis of acute intoxication (F10.0), urban area cases represent a majority of 338 cases $(65.6 \%)$ compared to rural areas that present a total of 177 cases $(34.4 \%)$. For the diagnosis of harmful alcohol use (F10.1), the urban environment represents a percentage of $67.9 \%$ compared to the rural environment which represents only $32.1 \%$.

Out of the total of 319 cases $(33.3 \%)$ from the rural area who were hospitalized in the mentioned period, $55.5 \%$ presented the diagnosis of F10.0 and $44.5 \%$ presented the diagnosis of (F10.1). On the other hand, in relation to patients from urban areas, and out of a total of 628 cases $(66.7 \%)$, the diagnosis of F10.0 was present at a percentage of $53.0 \%$, and the diagnosis of (F10.1) was present at a percentage of $47 \%$.

The number of patients from urban area were twice the number of patients coming from rural areas, as was observed in hospitalizations in the period of the pandemic, but the distribution according to the diagnosis between them being almost the same in both urban and rural areas.

\subsection{Gender and Diagnosis Distribution}

Table 2. Distribution by Gender and Diagnosis

\begin{tabular}{ccccccccc}
\hline Gender & \multicolumn{3}{c}{ F10.0 } & \multicolumn{3}{c}{ F10.1 } & \multicolumn{2}{c}{ TOTAL } \\
\hline & Freq & Col $\%$ & Row $\%$ & Freq & Col $\%$ & Row $\%$ & Freq & Col $\%$ \\
F & 60 & $11.7 \%$ & $57.7 \%$ & 44 & $10.0 \%$ & $42.3 \%$ & 104 & $10.9 \%$ \\
M & 455 & $88.3 \%$ & $53.3 \%$ & 398 & $90.0 \%$ & $46.7 \%$ & 853 & $89.1 \%$ \\
TOTAL & 515 & $100.0 \%$ & $53.8 \%$ & 442 & $100.0 \%$ & $46.2 \%$ & 957 & $100.0 \%$ \\
\hline
\end{tabular}

Source: Authors' own conception

During the analysed period, out of the total number of cases, 853 $(89.1 \%)$ were males and only $104(10.9 \%)$ were females. This confirms the 
increased consumption of alcohol among men and their ignorance of the imposed measures during the quarantine period.

Among males, 455 patients (53.8\%) had a diagnosis of F10.0 and 398 patients $(46.7 \%)$ had a diagnosis of (F10.1). Among females, 60 patients $(57.7 \%)$ had a diagnosis of F10.0 and 44 patients (42.3\%) had a diagnosis of (F10.1). Regarding these percentages, we can observe an increasing tendency of females to present at the psychiatry services for acute intoxication.

\subsection{Distribution according to Environment and Gender}

Table 3. Distribution by Environment and Gender

\begin{tabular}{ccccccccc}
\hline Environment & \multicolumn{3}{c}{$\mathrm{F}$} & & \multicolumn{3}{c}{$\mathrm{M}$} & \multicolumn{2}{c}{ TOTAL } \\
\hline & Freq & Col $\%$ & Row $\%$ & Freq & Col $\%$ & Row $\%$ & Freq & Col $\%$ \\
Rural & 23 & $22.1 \%$ & $7.2 \%$ & 296 & $34.7 \%$ & $92.8 \%$ & 319 & $33.3 \%$ \\
Urban & 81 & $77.9 \%$ & $12.7 \%$ & 557 & $65.3 \%$ & $87.3 \%$ & 638 & $66.7 \%$ \\
TOTAL & 104 & $100.0 \%$ & $10.9 \%$ & 853 & $100.0 \%$ & $89.1 \%$ & 957 & $100.0 \%$ \\
\hline
\end{tabular}

Source: Authors' own conception

In urban areas, males represent $87.3 \%$ (557 patients) compared to females $12.7 \%$ (81 patients). In rural areas, although the absolute number of cases was lower (319 patients), the male gender predominates in a higher percentage with $92.8 \%$ (296 patients) compared to the female gender with a percentage of $7.2 \%$ (23 patients).

There is a predominant percentage of males over females, both in urban areas $557(87.3 \%)$ and in rural areas $296(92.8 \%)$.

In the case of females, with only 104 cases, there is a strong predominance of the urban environment with $81(77.9 \%)$ compared to the rural area $23(22.1 \%)$. In men, there is a lower predominance of urban 557 $(65.3 \%)$ than in rural areas with $296(34.7 \%)$.

\subsection{Distribution by Month and Gender}

Table 4. Distribution by Month and Gender

\begin{tabular}{ccccccccc}
\hline Month & \multicolumn{2}{c}{$\mathrm{F}$} & \multicolumn{3}{c}{$\mathrm{M}$} & \multicolumn{2}{c}{ TOTAL } \\
\hline & Freq & Col $\%$ & Row $\%$ & Freq & Col $\%$ & Row $\%$ & Freq & Col $\%$ \\
January & 10 & $9.6 \%$ & $9.7 \%$ & 93 & $10.9 \%$ & $90.3 \%$ & 103 & $10.8 \%$ \\
February & 6 & $5.8 \%$ & $7.4 \%$ & 75 & $8.8 \%$ & $92.6 \%$ & 81 & $8.5 \%$ \\
March & 8 & $7.7 \%$ & $7.8 \%$ & 94 & $11.0 \%$ & $92.2 \%$ & 102 & $10.7 \%$ \\
April & 6 & $5.8 \%$ & $10.7 \%$ & 50 & $5.9 \%$ & $89.3 \%$ & 56 & $5.9 \%$ \\
May & 11 & $10.6 \%$ & $15.9 \%$ & 58 & $6.8 \%$ & $84.1 \%$ & 69 & $7.2 \%$ \\
June & 17 & $16.3 \%$ & $23.0 \%$ & 57 & $6.7 \%$ & $77.0 \%$ & 74 & $7.7 \%$ \\
July & 7 & $6.7 \%$ & $10.1 \%$ & 62 & $7.3 \%$ & $89.9 \%$ & 69 & $7.2 \%$
\end{tabular}




\begin{tabular}{ccccccccc} 
August & 11 & $10.6 \%$ & $14.7 \%$ & 64 & $7.5 \%$ & $85.3 \%$ & 75 & $7.8 \%$ \\
September & 4 & $3.8 \%$ & $5.9 \%$ & 64 & $7.5 \%$ & $94.1 \%$ & 68 & $7.1 \%$ \\
October & 10 & $9.6 \%$ & $10.5 \%$ & 85 & $10.0 \%$ & $89.5 \%$ & 95 & $9.9 \%$ \\
November & 9 & $8.7 \%$ & $11.4 \%$ & 70 & $8.2 \%$ & $88.6 \%$ & 79 & $8.3 \%$ \\
December & 5 & $4.8 \%$ & $5.8 \%$ & 81 & $9.5 \%$ & $94.2 \%$ & 86 & $9.0 \%$ \\
TOTAL & 104 & $100.0 \%$ & $10.9 \%$ & 853 & $100.0 \%$ & $89.1 \%$ & 957 & $100.0 \%$ \\
\hline
\end{tabular}

Source: Authors' own conception

Compared to the total number of cases in the examined period, it is noted that the months with the highest number of cases are: January with 103 (10.8\%), March with $102(10.7 \%)$ and October with 95 (9.9\%), and the months with the lowest number of cases are: April with 56 (5.9\%), September with $68(7.1 \%)$ and July with $69(7.2 \%)$. A model correlated with governmental measures related to the COVID-19 pandemic could not be established.

Regarding the female gender, we can see that the months with the highest number of cases are: June with 17 (16.3\%), August with $11(10.6 \%)$ and May with $11(10.6 \%)$, and the months with the lowest number of cases are: September with 4 (3.8\%), April and February with $6(5.8 \%)$. A model correlated with governmental measures related to the COVID-19 pandemic could not be established.

Compared to the male gender, it is observed that the months with the highest number of cases are: March with 94 (11.0\%), January with 93 $(10.9 \%)$ and October with $85(10.0 \%)$, and the months with the lowest number of cases were: April with 50 (5.9\%), June with 57 (6.7\%) and May with $58(6.8 \%)$.

\subsection{Distribution by month and Environment}

Table 5. Distribution by Month and Environment

\begin{tabular}{ccccccccc}
\hline Month & \multicolumn{3}{c}{ Rural } & \multicolumn{3}{c}{ Urban } & \multicolumn{2}{c}{ TOTAL } \\
\hline & Freq & Col $\%$ & Row $\%$ & Freq & Col $\%$ & Row $\%$ & Freq & Col $\%$ \\
January & 34 & $10.7 \%$ & $33.0 \%$ & 69 & $10.8 \%$ & $67.0 \%$ & 103 & $10.8 \%$ \\
February & 22 & $6.9 \%$ & $27.2 \%$ & 59 & $9.2 \%$ & $72.8 \%$ & 81 & $8.5 \%$ \\
March & 33 & $10.3 \%$ & $32.4 \%$ & 69 & $10.8 \%$ & $67.6 \%$ & 102 & $10.7 \%$ \\
April & 19 & $6.0 \%$ & $33.9 \%$ & 37 & $5.8 \%$ & $66.1 \%$ & 56 & $5.9 \%$ \\
May & 22 & $6.9 \%$ & $31.9 \%$ & 47 & $7.4 \%$ & $68.1 \%$ & 69 & $7.2 \%$ \\
June & 28 & $8.8 \%$ & $37.8 \%$ & 46 & $7.2 \%$ & $62.2 \%$ & 74 & $7.7 \%$ \\
July & 25 & $7.8 \%$ & $36.2 \%$ & 44 & $6.9 \%$ & $63.8 \%$ & 69 & $7.2 \%$ \\
August & 31 & $9.7 \%$ & $41.3 \%$ & 44 & $6.9 \%$ & $58.7 \%$ & 75 & $7.8 \%$ \\
September & 16 & $5.0 \%$ & $23.5 \%$ & 52 & $8.2 \%$ & $76.5 \%$ & 68 & $7.1 \%$
\end{tabular}




\begin{tabular}{ccccccccc}
\hline & & & & & & & & \\
October & 43 & $13.5 \%$ & $45.3 \%$ & 52 & $8.2 \%$ & $54.7 \%$ & 95 & $9.9 \%$ \\
November & 23 & $7.2 \%$ & $29.1 \%$ & 56 & $8.8 \%$ & $70.9 \%$ & 79 & $8.3 \%$ \\
December & 23 & $7.2 \%$ & $26.7 \%$ & 63 & $9.9 \%$ & $73.3 \%$ & 86 & $9.0 \%$ \\
TOTAL & 319 & $100.0 \%$ & $33.3 \%$ & 638 & $100.0 \%$ & $66.7 \%$ & 957 & $100.0 \%$ \\
\hline
\end{tabular}

Source: Authors' own conception

In relation to the urban area patients, it was observed that the months with the highest number of presentations were: January with 69 patients (10.8\%), March with 69 patients (10.8\%) December with 63 patients $(9.9 \%)$, and the months with the lowest number of presentations were: April 37 (5.8\%), July with 44 (6.9\%) and August with 44 (6.9\%).

In the urban environment, there is an association between the lowest number of presentations and the onset of the pandemic, we also notice the association between the highest number of presentations and the relaxation of measures before wave 2 of the pandemic.

In rural areas, the months with the highest number of presentations were: October with 43 patients (13.5\%), January with 34 patients $(10.7 \%)$ and March with 33 patients (10.3\%). While the months with the least presentations are: September with $16(5.0 \%)$, April with 19 patients $(6.0 \%)$ and May with 22 patients $(6.9 \%)$. The model that was marked in the rural areas seems to be a model related to the harvest and the specific holidays.

\subsection{Distribution by Gender and Day of the week}

Table 6. Distribution by Gender and Day of the week

\begin{tabular}{ccccccccc}
\hline Week day & \multicolumn{3}{c}{$\mathrm{F}$} & & \multicolumn{3}{c}{$\mathrm{M}$} & \multicolumn{3}{c}{ TOTAL } \\
\hline & Freq & Col $\%$ & Row $\%$ & Freq & Col $\%$ & Row $\%$ & Freq & Col $\%$ \\
Monday & 23 & $22.1 \%$ & $12.9 \%$ & 155 & $18.2 \%$ & $87.1 \%$ & 178 & $18.6 \%$ \\
Thursday & 15 & $14.4 \%$ & $13.9 \%$ & 93 & $10.9 \%$ & $86.1 \%$ & 108 & $11.3 \%$ \\
Wednesday & 8 & $7.7 \%$ & $6.3 \%$ & 118 & $13.8 \%$ & $93.7 \%$ & 126 & $13.2 \%$ \\
Tuesday & 17 & $16.3 \%$ & $13.5 \%$ & 109 & $12.8 \%$ & $86.5 \%$ & 126 & $13.2 \%$ \\
Friday & 21 & $20.2 \%$ & $13.7 \%$ & 132 & $15.5 \%$ & $86.3 \%$ & 153 & $16.0 \%$ \\
Saturday & 11 & $10.6 \%$ & $8.3 \%$ & 121 & $14.2 \%$ & $91.7 \%$ & 132 & $13.8 \%$ \\
Sunday & 9 & $8.7 \%$ & $6.7 \%$ & 125 & $14.7 \%$ & $93.3 \%$ & 134 & $14.0 \%$ \\
TOTAL & 104 & $100.0 \%$ & $10.9 \%$ & 853 & $100.0 \%$ & $89.1 \%$ & 957 & $100.0 \%$ \\
\hline
\end{tabular}

Source: Authors' own conception

For the female gender, it was observed that the days with the highest number of presentations were: Monday with 23 patients (22.1\%) and Friday with 21 patients $(20.2 \%)$, and the days with the least presentations were: Wednesday with 8 patients $(7.7 \%)$ and Sunday with 9 patients $(8.7 \%)$. 
For males, the days with the highest number of presentations were: Monday with 155 patients (18.2\%) and Friday with 132 patients (15.5\%), and the days with the least presentations were Tuesday with 93 patients $(10.9 \%)$ and Thursday with 109 patients (12.8\%).

We notice a concordance related to Mondays and Fridays in both genders.

\subsection{Distribution by Month and Diagnosis}

Table 7. Distribution by Month and Diagnosis

\begin{tabular}{ccccccccc}
\hline Month & \multicolumn{3}{c}{ F10.0 } & \multicolumn{3}{c}{ F10.1 } & \multicolumn{2}{c}{ TOTAL } \\
\hline January & 62 & $12.0 \%$ & $60.2 \%$ & 41 & $9.3 \%$ & $39.8 \%$ & 103 & $10.8 \%$ \\
February & 35 & $6.8 \%$ & $43.2 \%$ & 46 & $10.4 \%$ & $56.8 \%$ & 81 & $8.5 \%$ \\
March & 57 & $11.1 \%$ & $55.9 \%$ & 45 & $10.2 \%$ & $44.1 \%$ & 102 & $10.7 \%$ \\
April & 27 & $5.2 \%$ & $48.2 \%$ & 29 & $6.6 \%$ & $51.8 \%$ & 56 & $5.9 \%$ \\
May & 39 & $7.6 \%$ & $56.5 \%$ & 30 & $6.8 \%$ & $43.5 \%$ & 69 & $7.2 \%$ \\
June & 48 & $9.3 \%$ & $64.9 \%$ & 26 & $5.9 \%$ & $35.1 \%$ & 74 & $7.7 \%$ \\
July & 33 & $6.4 \%$ & $47.8 \%$ & 36 & $8.1 \%$ & $52.2 \%$ & 69 & $7.2 \%$ \\
August & 51 & $9.9 \%$ & $68.0 \%$ & 24 & $5.4 \%$ & $32.0 \%$ & 75 & $7.8 \%$ \\
September & 33 & $6.4 \%$ & $48.5 \%$ & 35 & $7.9 \%$ & $51.5 \%$ & 68 & $7.1 \%$ \\
October & 39 & $7.6 \%$ & $41.1 \%$ & 56 & $12.7 \%$ & $58.9 \%$ & 95 & $9.9 \%$ \\
November & 43 & $8.3 \%$ & $54.4 \%$ & 36 & $8.1 \%$ & $45.6 \%$ & 79 & $8.3 \%$ \\
December & 48 & $9.3 \%$ & $55.8 \%$ & 38 & $8.6 \%$ & $44.2 \%$ & 86 & $9.0 \%$ \\
TOTAL & 515 & $100.0 \%$ & $53.8 \%$ & 442 & $100.0 \%$ & $46.2 \%$ & 957 & $100.0 \%$ \\
\hline
\end{tabular}

Source: Authors' own conception

The months with the highest number of presentations for the diagnosis of acute intoxication (F10.0) were: January with 62 patients $(12.0 \%)$ and March with 57 patients (11.1\%), and the months with the lowest presentations were: April with 27 patients (5.2\%) and July and September with 33 patients $(6.4 \%)$.

For the diagnosis of (F10.1), the months with the highest number of presentations were: October with 56 patients (12.7\%) and February with 46 patients $(10.4 \%)$, and the months with the least presentations were: August with 24 patients (5.4\%) and April with 29 patients (6.6\%)

April had the lowest number of hospitalizations regarding both diagnoses, most likely, due to the measures imposed in relation to COVID19. 


\section{Conclusions}

During the analysed period, there were 7614 discharges from the 'Elisabeta Doamna' Psychiatriy Hospital, of which 1465 patients (20.08\%) diagnosed with disorders related to alcohol consumption. Disorders related to intoxication and alcohol use represent a percentage of $13.14 \%$ (957 cases) of the total discharges, and $65.46 \%$ of the total disorders related to alcohol.

Harmful use disorders (F10.1) represent a percentage of 30.31\% (442 cases) of the total alcohol-related disorders. On the other hand, disorders due to acute intoxication (F10.0), represented a percentage of $35.15 \%$ (515 cases).

After analysing the data, the following points were observed:

- those in urban areas predominate by a percentage of $66.7 \%$

- cases with (F10.0) predominate by a percentage of 53.8\%

- male predominates by a percentage of $89.1 \%$

From the view of the dispersion of the number of cases, there is a fluctuation in the tendency to follow the restrictions imposed by the authorities.

\section{References}

American Psychiatric Association. (2013). Diagnostic and statistical manual of mental disorders (5th ed.). American Psychiatric Publishing.

Ciubara, A. B., Tudor, R. C., Nechita, L., Tita, O., Ciubara, A., Turliuc, S., \& Raftu, G. (2018). The composition of bioactive compounds in wine and their possible influence on osteoporosis and on bone consolidation. Revista de Chimie, 69(5), 1247-1253. https://doi.org/10.37358/rc.18.5.6300

Ciubara, A., Burlea, S. L., Sacuiu, I., Radu, D. A., Untu, I., \& Chirita, R. (2015). Alcohol addiction - a psychosocial perspective. Procedia Social and Behavioral Sciences, 187, 536-540. https://doi.org/10.1016/j.sbspro.2015.03.100

Luca, L., Baroiu, L., Ciubara, A. B., Anghel, R., Bulgaru-Iliescu, A. I., Anghel, L., \& Ciubara, A. (2020). Covid-19 and the Spanish Flu. From suffering to resilience, BRAIN. Broad Research in Artificial Intelligence and Neuroscience, 11(3S1), 01-07. https://doi.org/10.18662/brain/11.3sup1/116

Luca, L., Ciubara, A. B., Fulga, I., Burlea, S. L., Terpan, M., \& Ciubara, A. M. (2020). Social implications for psychiatric pathology of depressive and anxiety disorders, alcohol addiction and psychotic disorders during the COVID-19 pandemic in Romania. Analysis of two relevant psychiatry hospitals. Revista de Cercetare si Interventie Sociala, 69, 261-272. https://doi.org/10.33788/rcis.69.16 
Sandu, A. (2020a). Bioethics - Philosophy or Science?. Logos Universality Mentality Education Novelty: Philosophy \& Humanistic Sciences, 8(1), 44-60. https://doi.org/10.18662/lumenphs/8.1/35

Sandu, A. (2020b). Bioethics of Public Policies. Ethical Standards in Crisis Situations. Postmodern Openings, 11(1Sup2), 141-160. https://doi.org/10.18662/po/11.1sup2/147

Sandu, A. (2020c). Pandemic - Catalyst of the Virtualization of the Social Space. Postmodern Openings, 11(1Sup2), 115-140. https://doi.org/10.18662/po/11.1sup2/146

SOFA. (n.d.). Statistics open for all, version 1.5.4. bttps:// wmw.sofastatistics.com/ home.php

Tabian, D., Drochioiu, G., Damian, S-I., Girlescu, N., Toma Gradinaru, O., Toma, S. I., \& Bulgaru-Iliescu, D. (2021). Toxic Blood Hydrogen Cyanide Concentration as a Vital Sign of a Deceased Room Fire Victim-Case Report. Toxics, 9(2), 36. http://dx.doi.org/10.3390/toxics9020036

The jamovi project. (2021). https://www.jamovi.org

World Health Organization. (2016). International statistical classification of diseases and related health problems (10th ed.). World Health Organization. 